\title{
COMPRESSED SENSING OF COMPLEX SINUSOIDS OFF THE GRID
}

\author{
Cheng Ping $^{*}$ — Shi Liu ${ }^{*}$ - Zhao Jiaqun ${ }^{* *}$
}

\begin{abstract}
To solve off-grid problem in compressed sensing, a new reconstruction algorithm for complex sinusoids is proposed. The compressed sensing reconstruction problem is transformed into a joint optimized problem. Based on coordinate descent approach and linear estimator, a new iteration algorithm is proposed. The results of experiments verify the effectiveness of the proposed method.
\end{abstract}

K e y w or d s: compressed sensing, off-grid, complex sinusoids, coordinate descent, linear estimator

\section{INTRODUCTION}

Compressed sensing (CS) has received much attention in wide application recently. As complex sinusoids signal model is widely used in application, research of CS for complex sinusoids is very important. However parameter discretization brings off-gird problem in compressed sensing, which makes its performance degrade significantly. In 2011, Y. Chi studied the sensitivity of Basis Pursuit (BP) algorithm to off the grid, and pointed out it needs more consideration in application [1]. D. H. Chae proposed that building up CS basis element more finely can reduce the effect of off-grid [2]. However, the method needs more reconstruction time and more measurements. To decrease the computational complexity, the grid can be refined only around the regions where sources are present [3]. For widely separated objects, A. F. Jiang proposed a method based on band exclusion and local optimization [4]. Under some special conditions, the methods proposed in $[5,6]$ can be used. M.F. Duarte has proposed spectral compressed sensing (SCS) to solve off-grid problem [7]. L. Hu proposed a method based on basis refinement for complex sinusoids [8], which has better performance than SCS. As the computational cost is very high, L. Hu then developed a fast and accurate reconstruction algorithm [9], which applies a liner approximation to the true unknown dictionary. G. Tang has investigated compressed sensing off the gird and proposed an atomic norm minimization approach [10]. But it is provided that the frequencies are well separated. In this paper, a novel compressed sensing reconstruction algorithm for complex sinusoids is proposed, which selects dictionary adaptively according signal and can eliminate off-grid problem. Compared with the existing methods, the proposed algorithm has several advantages. The first advantage is that it is very simple and has low computational cost. The second advantage is that it does not require that the frequencies are well separated. The third advantage is that it can achieve high reconstruction accuracy.

\section{THE PROPOSED METHOD}

Supposing signal $x_{n}$ can be modeled as a superposition of $K$ complex sinusoids

$$
x_{n}=\sum_{i=1}^{K} s_{i} e^{-j 2 \pi f_{i} n}, \quad n=0,1, \ldots, N-1
$$

where $f_{i} \in[0,1)$ is the $i$-th component normalized frequency and $s_{i} \in C$ is the corresponding complex amplitude. Let $f=\left[f_{1}, f_{2}, \ldots, f_{K}\right]^{\top}, s=\left[s_{1}, s_{2}, \ldots, s_{K}\right]^{\top}$, $A(f)=\left[b\left(f_{1}\right) b\left(f_{2}\right) \ldots b\left(f_{K}\right)\right]$ and $b\left(f_{i}\right)=\left[e^{-j 2 \pi f_{i} 0}\right.$, $\left.e^{-j 2 \pi f_{i} 1}, \ldots, e^{-j 2 \pi f_{i}(N-1)}\right]^{\top},(1)$ can be expressed as

$$
x=A(f) s .
$$

In compressed sensing, supposing $\Phi$ is measurement matrix of size $M \times N$,measurement $y$ can be expressed as

$$
y=\Phi x+\varepsilon
$$

where $y=\left[y_{0}, y_{1}, \ldots, y_{M-1}\right]^{\top}$ and $\varepsilon=\left[\varepsilon_{0}, \varepsilon_{1}, \ldots, \varepsilon_{M-1}\right]^{\top}$ is noise.

As frequency components of $x$ are not known, a predefined Fourier dictionary is adopted in compressed sensing. For an arbitrary frequency grid $f=\left[f_{1}, f_{2}, \ldots, f_{P}\right] \in$ $[0,1)^{P}$ (where $P$ is the number of grids), the corresponding Fourier dictionary is $D(f)=\left[b\left(f_{1}\right) b\left(f_{2}\right) \ldots b\left(f_{P}\right)\right]$. When there exist off-grid frequencies, the performance of CS degrades greatly, which is called off-grid problem.

College of Information and Communication Engineering, Harbin Engineering University, Harbin 150001, People's Republic of China, chengping1219@126.com ${ }^{* *}$ College of Science, Hohai University, Nanjing 211100, Republic of China, jqzhao_03@163.com 
If a frequency grid $f_{S}=\left[f_{S 1}, f_{S 2}, \ldots, f_{S P}\right]$ contains all component frequencies of $x$, there is no off-grid problem. Let $D\left(f_{S}\right)$ be the Fourier dictionary corresponding to $f_{S}$ and $a$ be a vector of sparse representation coefficients of $x$ with respect to $D\left(f_{S}\right)$, ie $x=D\left(f_{S}\right) a$. The measurement model (3) can be reformulated as

$$
y=\Phi D\left(f_{S}\right) a+\varepsilon .
$$

Thus, the problem to reconstruct $x$ is to recover $f_{S}$ and $a$ from $y$ based on the model given in (4), which is to minimize the following cost function

$$
J\left(a, f_{s}\right)=\left\|y-\Phi D\left(f_{s}\right) a\right\|_{2}^{2}+\lambda\|a\|_{p}^{p}, \quad 0<p \leq 1 .
$$

To get the joint minimization, a coordinate descent approach can be employed, which optimizes dictionary parameters and sparse coefficients alternately

$$
\begin{aligned}
f_{s}^{(t+1)} & =\arg \min _{f_{s}}\left\|y-\Phi D\left(f_{s}\right) a^{(t)}\right\|_{2}^{2}+\lambda\left\|a^{(t)}\right\|_{p}^{p} \\
& =\arg \min _{f_{s}}\left\|y-\Phi D\left(f_{s}\right) a^{(t)}\right\|_{2}^{2} \\
a^{(t+1)} & =\arg \min _{a}\left\|y-\Phi D\left(f_{s}^{(t+1)}\right) a\right\|_{2}^{2}+\lambda\|a\|_{p}^{p}
\end{aligned}
$$

where $t$ is the number of iterations.

However when there is mismatch between $f_{s}^{(t)}$ and $f_{S}$, there is big error in $a^{(t)}$. In the above iteration, the estimation of $f_{s}^{(t+1)}$ is based on $a^{(t)}$, and then the estimation of $a^{(t+1)}$ is based on $f_{s}^{(t+1)}$. Therefore the above iteration can not guarantee convergence to $f_{S}$ and $a$.

In [11], the result of CS is improved by providing more accurate amplitude and phase estimates using linear estimator. Inspired by this idea, a novel compressed sensing reconstruction algorithm is proposed, which has the following steps:

(1) Initial frequency estimation: Let $t=1$. A conventional compressed sensing algorithm (such as BP, FOCUSS [12], or Matching Pursuit) is used to get initial frequency location $f_{s}^{(1)}$ and sparse representation coefficients $a^{(0)}$. Adaptive gird refinement can be used here [3]. As some of frequencies are off the grid, the amplitude estimation is not correct.

(2) Amplitude estimation: Only selecting the columns corresponding to $K$ local maximum of the nonzero elements of $a^{(t-1)}$, the matrix $\Phi D\left(f_{s}^{(t)}\right)$ of size $M \times P$ is scaled down to much slimmer matrix $R$ of size $M \times$ $K$. Thus the original underdetermined problem becomes overdetermined [11]

$$
y=R \gamma+\varepsilon
$$

where $\gamma$ is complex amplitude for the $K$ frequency components of $a^{(t)}$.

The amplitude $\gamma$ is finally found by [11]

$$
\gamma=\left(R^{H} R\right)^{-1} R^{H} y
$$

where $R^{H}$ denotes conjugate transpose of $R$.

Then $a^{(t)}$ can be obtained: values in local maximum locations are set by $\gamma$ and other values are set by zeros.

(3) Accurate frequency estimation: $f_{s}^{(t+1)}$ is obtained based on (6).

(4) Accurate amplitude estimation: Let $t=t+1$. Do step (2) to get accurate complex amplitude estimation $a^{(t)}$.

(5) Repeat step (3) and (4) until estimation accuracy is satisfied.

In the above algorithm, step (2) supplies more accurate complex amplitude for CS by using linear estimator. So only executing step (1) and step (2), the estimation performance can also be improved. A method which consists of step (1) and step (2) is called Modi-CS in the following. Step $(3) \sim(5)$ is a modified coordinate descent approach, which can improve the estimation performance further.

\section{EXPERIMENTAL RESULTS AND DISCUSSION}

Numerical experiments are done to verify the effectiveness of the proposed method. In the experiments, signal $x$ with length $N=320$ contains $K=5$ complex sinusoids. Their normalized frequencies and amplitudes are distributed randomly over $[0,1)$ and the complex unit circle respectively. Measurement matrix $\Phi$ is random Gauss matrix, and a length $M=64$ measurement is obtained according (3). The measurement noise level is measured by "peak signal-to-noise ratio" (PSNR), which is defined as $\mathrm{PSNR}=10 \log _{10}\left(1 / \sigma^{2}\right)$, where $\sigma^{2}$ denotes the variance of the measurement noise. The PSNR is varied from $5 \mathrm{~dB}$ to $45 \mathrm{~dB}$. For each PSNR, 100 independent trials are executed. Reconstruction accuracy is measured by the "reconstruction signal-to-noise ratio" (RSNR), which is defined as

$$
\mathrm{RSNR}=20 \log _{10}\left(\|x\|_{2} /\|x-\hat{x}\|_{2}\right)
$$

where $\hat{x}$ is the estimate of $x$. Average RSNRs of different PSNR levels are shown in Fig. 1 for CS, Modi-CS and the proposed method. Here FOCUSS is used as a CS method and it is used in step (1) in Modi-CS and the proposed method.

It can be seen that the proposed method always outperforms the other two methods whenever the PSNR is high or small. As the frequencies are randomly selected, for any predefined Fourier dictionary, there are off-grid frequencies. So the performance of CS degrades significantly. As the proposed method can solve off-grid problem, its performance is much better. Furthermore, for the proposed method, the signal reconstruction accuracy and the noise level are approximately linear, which indicates the proposed method is robust to the noise in the measurements.

A wide range of numerical experiments demonstrated that the proposed algorithm can reconstruct signals with 


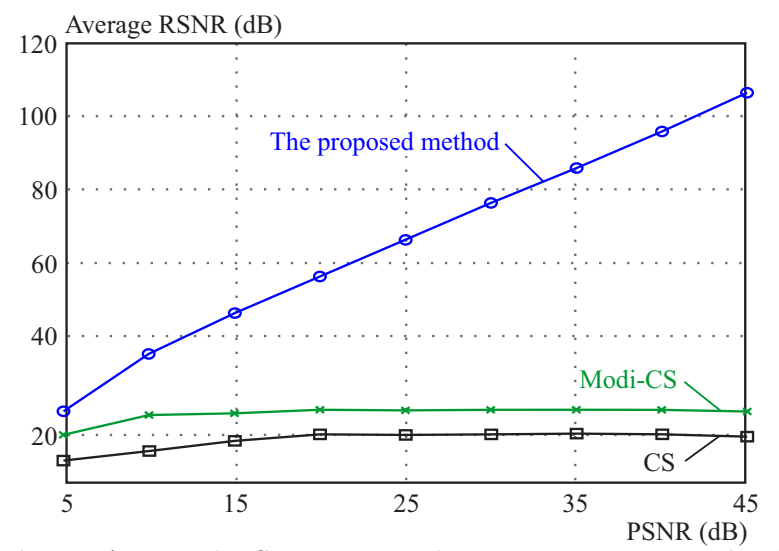

Fig. 1. Averaged RSNRs versus the measurement noise level

closely-space component frequencies. Although we take complex sinusoids as the preferred application, the proposed algorithm is applicable to other signals.

\section{CONCLUSION}

To solve off-grid problem in compressed sensing of complex sinusoids, a novel reconstruction method is proposed. Compressed sensing problem is recast as a joint minimization problem. A novel iterative algorithm is proposed to get the joint minimization. The effectiveness of the method is verified by experiments.

In some applications, such as DOA estimation, ISAR (inverse synthetic aperture radar) imaging and tomographic SAR, high-quality compressed sensing reconstruction is needed. However compressed sensing can not supply high quality reconstruction in presence of off-grid. The proposed method has supplied a simple and effective method to eliminate off-grid problem in compressed sensing. The algorithm does not require that the frequencies are widely separated. It not only can be used for complex sinusoids but also can be used for other signals.

\section{Acknowledgments}

This work was supported by National Natural Science Foundation of China (grant No. 61301199), Research Fund for the Doctoral Program of Higher Education of China (grant No. 20122304120017), and the Fundamental Research Funds for the Central Universities (grant No. HEUCF150815).

\section{REFERENCES}

[1] CHI, Y.-SCGARF, L. L.-PEZESHKI, A.-CALDERBANK, A. R.: Sensitivity to Basis Mismatch in Compressed Sensing, IEEE Trans. Signal Process. 59 No. 5 (2011), 2182-2195.
[2] CHAE, D. H.-SADEGHI, P.-KEnNEDY, R. A.: Effects of Basis-Mismatch in Compressive Sampling of Continuous Sinusoidal Signals, $2^{\text {nd }}$ Int. Conf. Future Comput. Commun., Wuhan, China, May 2010, pp. 739-743.

[3] Malioutov, D.-Cetin, M.-Willsky, A. S. : A Sparse Signal Reconstruction Perspective for Source Localization with Sensor Arrays, IEEE Trans. Signal Process. 53 No. 8 (2005), 3010-3022.

[4] FAngJiAnG, A.-LIAO, W.: Coherence Pattern-Guided Compressive Sensing with Unresolved Grids, SIAM J. Imag. Sci. 5 No. 1 (2012), 179-202.

[5] ZHU, H.-LEUS, G.-GIANNAKIS, G. B.: Sparsity-Congnizant Total Least-Squares for Perturbed Compressive Sampling, IEEE Trans. Signal Process. 59 No. 5 (2011), 2002-2016.

[6] YANG, Z.-ZHANG, C.-XIE, L. : Robustly Stable Signal Recovery in Compressed Sensing with Structured Matrix Perturbation, IEEE Trans. Signal Process. 60 No. 9 (2012), 4658-4671.

[7] DUARTe, M. F.-BARAniUK, R. G.: Spectral Compressive Sensing, Appl. Comput. Harmon. Anal. 35 No. 1 (2013), 111-129.

[8] HU, L.-SHI, Z.-ZHOU, J.-FU, Q. : Compressed Sensing of Complex Sinusoids: an Approach based on Dictionary Refinement, IEEE Trans. Signal Process. 60 No. 7 (2012), 3809-3822.

[9] HU, L.-ZHOU, J.-SHI, Z.-FU, Q.: A Fast and Accurate Reconstruction Algorithm for Compressed Sensing of Complex Sinusoids, IEEE Trans. Signal Process. 61 No. 22 (2013), $5744-5754$.

[10] TANG, G.-BHASKAR, B. N.-SHAH, P.-RECHT, B. : Compressed Sensing off the Grid, IEEE Trans. Inf. Theory 59 No. 11 (2013), 7465-7490.

[11] ZHU, X. X.-BAMLER, R. : Super-Resolution Power and Robustness of Compressive Sensing for Spectral Estimation with Application to Spaceborne Tomographic SAR, IEEE Trans. Geosci. Remote Sens. 50 No. 1 (2011), 247-258.

[12] GORODNITSKY, I. F.-RAO, B. D.: Sparse Signal Reconstruction from Limited Data using FOCUSS: A Re-weighed Minimum Norm Algorithm, IEEE Trans. Signal Process. 45 No. 3 (1997), 600-616.

Received 11 November 2014

Cheng Ping was born in Jiangsu, China, in 1976. She received Bachelor Degree and Master Degree in electronic engineering from Harbin Institute of Technology in 2000 and 2002 respectively, and $\mathrm{PhD}$ degree in the field of communication and information system in Harbin Institute of Technology in 2007. She has been working in Harbin Engineering University since 2007. Her research interest is compressed sensing, inverse synthetic aperture radar (ISAR) imaging. She has published nearly 30 journals and conference papers.

Shi Liu was born in Hebei, China, in 1990. She is currently pursuing Master Degree in information and communication system in Harbin Engineering University, China. Her current research is focused on modified compressive sensing.

Zhao Jiaqun was born in Heilongjiang, China, in 1976. He received Master Degree in optics and PhD degree in physical electronics from Harbin Institute of Technology in 2003 and 2011, respectively. His research is mainly focused on solid state laser and nonlinear optical frequency conversion. 